\title{
Bone turnover markers indicate risk of fracture
}

Identifying women at high risk of fragility fractures is a challenge for the management of osteoporosis. Increased bone metabolism is a potential risk factor for fracture, and Ivaska et al. now provide evidence that elevated levels of markers of bone turnover are associated with an increased risk of fracture for nearly a decade.

The researchers measured the levels of seven markers of bone turnover in a population-based sample of 1,040 women aged 75 years at baseline and 1 year after baseline. During a mean 9-yearfollow-up, 35\% of the women sustained at least one fracture (of any type). High baseline levels of serum tartrate-resistant acid phosphatase $5 \mathrm{~b}$ (S-TRACP5b), serum concentrations of the C-terminal telopeptide of collagen I (S-CTX-I) and levels of urinary midfragments of osteocalcin were associated with an increased risk of any type of fracture except those of the hip (hazard ratio/SD = 1.16, 1.13 and 1.08 , respectively). Levels of markers of bone formation were not associated with the risk of fracture.

When assessed 1 year after the baseline measurement, high levels of S-TRACP5b and S-CTX-I were again associated with an increased fracture risk; an average of the measurements at baseline and after 1 year gave similar results in terms of fracture prediction.

The markers of bone turnover were mostly associated with fractures sustained at the beginning of the follow-up period; the association was particularly strong for vertebral fractures, with the predictive ability lasting for at least 5 years.

Katrin Legg

Original article Ivaska, K. K. et al. Bone turnover markers and prediction of fracture: a prospective follow-up study of 1040 elderly women for a mean of nine years. J. Bone Miner. Res. doi:10.1359/JBMR.091006 